TRANSACTIONS OF THE

AMERICAN MATHEMATICAL SOCIETY

Volume 348, Number 1, January 1996

\title{
A PROBLEM OF FÖLDES AND PURI ON THE WIENER PROCESS
}

\author{
Z. SHI
}

\begin{abstract}
Let $W$ be a real-valued Wiener process starting from 0 , and $\tau(t)$ be the right-continuous inverse process of its local time at 0 . Földes and Puri [3] raise the problem of studying the almost sure asymptotic behavior of $X(t)=\int_{0}^{\tau(t)} \mathbf{1}_{\{|W(u)| \leq \alpha t\}} d u$ as $t$ tends to infinity, i.e. they ask: how long does $W$ stay in a tube before "crossing very much" a given level? In this note, both limsup and liminf laws of the iterated logarithm are provided for $X$.
\end{abstract}

\section{INTRODUCTION}

Let $W$ be a real-valued Wiener process with $W(0)=0$. Define its local time process

$$
\ell(t, x)=\frac{d}{d x} \int_{0}^{t} \mathbb{1}_{\{W(u)<x\}} d u,
$$

which is almost surely continuous (up to a modification, which is taken for granted) in $(t, x)$ according to Trotter's theorem $([7])$. For a modern account of Wiener local times, see for example Revuz and Yor [6, Chap. VI] from stochastic analysis viewpoint, and Révész [5, Part I] through sample paths. We denote $\ell(t, 0)$ by $\ell(t)$ for notational convenience, and write

$$
\tau(s)=\inf \{t>0: \ell(t)>s\}, \quad s>0,
$$

the right-continuous inverse process of $\ell(t)$. In their recent paper on Wiener processes, Földes and Puri [3] raise the following problem: let

$$
X(t)=\int_{0}^{\tau(t)} \mathbb{1}_{\{|W(u)| \leq \alpha t\}} d u, \quad t>0,
$$

with a fixed constant $\alpha>0$, what can be said about the almost sure asymptotics of $X$ ?

Our answer to the "limsup" part of the question is a law of the iterated logarithm (LIL).

Theorem 1. For any $\alpha>0$,

$$
\limsup _{t \rightarrow \infty} \frac{X(t)}{t^{2} \log \log t}=\frac{8 \alpha^{2}}{\pi^{2}} \quad \text { a.s. }
$$

For the "liminf" behavior of $X$, we have an integral test characterizing its lower functions.

Received by the editors December 7, 1994.

1991 Mathematics Subject Classification. Primary 60J65; Secondary 60G17.

Key words and phrases. Wiener process (Brownian motion), law of the iterated logarithm.

(C)1996 American Mathematical Society 
Theorem 2. Let $\alpha>0$, and let $f>0$ be a non-decreasing function. Then

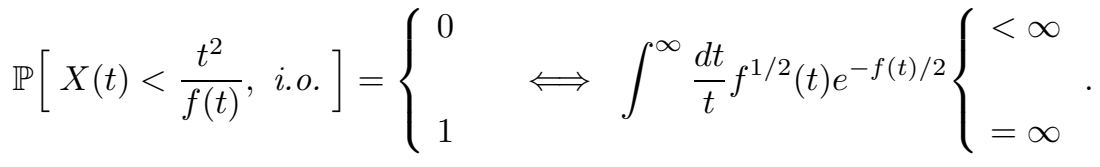

In particular, the following Chung-type LIL holds:

$$
\liminf _{t \rightarrow \infty} \frac{\log \log t}{t^{2}} X(t)=\frac{1}{2} \quad \text { a.s. }
$$

Remarks. (i) In (1.3), the symbol "i.o." means "infinitely often" as $t$ tends to infinity.

(ii) It is somewhat surprising that the lower functions of $X$ do not depend on the value of $\alpha$. Formally (i.e. informally!) taking $\alpha=\infty$ (thus $X(t)=\tau(t)$ ) in (1.3) and several lines of elementary calculation yield

$$
\mathbb{P}\left[\ell(t)>(t f(t))^{1 / 2}, \text { i.o. }\right]=\left\{\begin{array} { l } 
{ 0 } \\
{ 1 }
\end{array} \int ^ { \infty } \frac { d t } { t } f ^ { 1 / 2 } ( t ) e ^ { - f ( t ) / 2 } \left\{\begin{array}{l}
<\infty \\
=\infty
\end{array},\right.\right.
$$

for any non-decreasing function $f>0$. We therefore recover the Erdös-FellerKolmogorov-Petrov test for the upper functions of $W$, since the classical Lévy's identity asserts that $\{\ell(t) ; t \geq 0\}$ and $\left\{\sup _{0 \leq s \leq t} W(s) ; t \geq 0\right\}$ have the same law.

Theorem 1 is proved in Section 2, and Theorem 2 in Section 3.

\section{Proof of Theorem 1}

Let $X(t)$ be defined as in (1.1). It follows from Brownian scaling that

$$
X(t) \stackrel{(d)}{=} t^{2} X(1)
$$

for any $t>0$ (the symbol " $\stackrel{(d)}{=}$ " denoting identity in distribution). We are first interested in the Laplace transform of $X(1)$.

Lemma 1. For any $\theta>0$,

$$
\mathbb{E} \exp \left(-\frac{\theta^{2}}{2} X(1)\right)=\exp (-\theta \tanh (\alpha \theta)) .
$$

Remark. This has previously been obtained by Földes and Puri [3, p. 536] by solving the corresponding Sturm-Liouville equation. Our proof, based on the RayKnight theorem, allows us to make use of a Lévy-type formula, thus avoiding further computation.

Proof of Lemma 1. By occupation times formula for local times (see for example Revuz and Yor [6, Corollary VI.1.6]), $X(1)=\int_{-\alpha}^{\alpha} \ell(\tau(1), x) d x$. The Brownian excursion theory confirms the independence between the processes

$$
\{\ell(\tau(1), x), x \geq 0\} \text { and }\{\ell(\tau(1),-x), x \geq 0\},
$$


which, according to the second Ray-Knight theorem (Revuz and Yor [6, Theorem XI.2.3]), behave both like a square Bessel process of dimension 0 (i.e. a linear diffusion process with generator $2 x \frac{d^{2}}{d x^{2}}$, absorbed at 0 ), starting from 1 . Thus by scaling,

$$
\begin{aligned}
\mathbb{E} \exp \left(-\frac{\theta^{2}}{2} X(1)\right) & =\left[\mathbb{E} \exp \left(-\frac{\theta^{2}}{2} \int_{0}^{\alpha} R^{2}(x) d x\right)\right]^{2} \\
& =\left[\mathbb{E} \exp \left(-\frac{(\theta \alpha)^{2}}{2} \int_{0}^{1} \hat{R}^{2}(s) d s\right)\right]^{2},
\end{aligned}
$$

where $R$ and $\hat{R}$ are Bessel processes of dimension 0 , with $R(0)=1$ and $\hat{R}(0)=$ $1 / \sqrt{\alpha}$. Now Lemma 1 is a particular case (taken for dimension 0 ) of a formula for square Bessel processes presented in Revuz and Yor [6, Corollary XI.1.8].

Remark. For connections between quadratic functionals of Bessel processes and the Sturm-Liouville equation, see for example Pitman and Yor [4].

Although it does not seem trivial to deduce the exact upper tail of $X(1)$ from its Laplace transform (2.2), one can manage to obtain the following useful estimate.

Lemma 2. For any $\alpha>0$, we have

$$
\lim _{\lambda \rightarrow \infty} \frac{1}{\lambda} \log \mathbb{P}(X(1)>\lambda)=-\frac{\pi^{2}}{8 \alpha^{2}} .
$$

Proof of Lemma 2. We begin with the upper bound. Fix $0<\varepsilon<1$. From (2.2) it follows using analytic continuation that

$$
\mathbb{E} \exp \left(\frac{\theta^{2}}{2} X(1)\right)=\exp (\theta \operatorname{tg}(\alpha \theta))
$$

for $0<\theta<\pi / 2 \alpha$. Consequently, for $\lambda>0$, we have

$$
\begin{aligned}
\mathbb{P}(X(1)>\lambda) & \leq \exp \left(-\frac{\pi^{2}(1-\varepsilon)^{2}}{8 \alpha^{2}} \lambda\right) \mathbb{E} \exp \left(\frac{\pi^{2}(1-\varepsilon)^{2}}{8 \alpha^{2}} X(1)\right) \\
& =\exp \left(-\frac{\pi^{2}(1-\varepsilon)^{2}}{8 \alpha^{2}} \lambda+\frac{\pi(1-\varepsilon)}{2 \alpha} \operatorname{tg}\left(\frac{\pi(1-\varepsilon)}{2}\right)\right),
\end{aligned}
$$

which yields

$$
\limsup _{\lambda \rightarrow \infty} \frac{1}{\lambda} \log \mathbb{P}(X(1)>\lambda) \leq-\frac{\pi^{2}(1-\varepsilon)^{2}}{8 \alpha^{2}} .
$$

The upper bound is proved by sending $\varepsilon$ to $0^{+}$. For the lower bound, let

$$
\begin{aligned}
& H_{\alpha}=\inf \{t>0:|W(t)|=\alpha\} ; \\
& \sigma_{0}=\inf \left\{t>H_{\alpha}: W(t)=0\right\} .
\end{aligned}
$$

Observe that when $t \in\left(H_{\alpha}, \sigma_{0}\right)$, the Wiener process $W$ stays away from 0 , and thus $\ell(t)$ does not increase. Consequently, $\sigma_{0} \leq \tau(1)$ in case $H_{\alpha}<\tau(1)$. Define 
$\hat{W}(t)=W\left(t+H_{\alpha}\right)-W\left(H_{\alpha}\right)$ which is again a standard Wiener process, independent of $\left\{W(u) ; 0 \leq u \leq H_{\alpha}\right\}$. For any $x$, write $\hat{T}_{x}=\inf \{t>0: \hat{W}(t)=x\}$. Obviously,

$$
\begin{aligned}
X(1) \geq & \mathbb{1}_{\left\{H_{\alpha}<\tau(1)\right\}} \int_{H_{\alpha}}^{\sigma_{0}} \mathbb{1}_{\{|W(u)| \leq \alpha\}} d u \\
= & \mathbb{1}_{\left\{H_{\alpha}<\tau(1), W\left(H_{\alpha}\right)=-\alpha\right\}} \int_{0}^{\hat{T}_{\alpha}} \mathbb{1}_{\{\hat{W}(u) \geq 0\}} d u \\
& \quad+\mathbb{1}_{\left\{H_{\alpha}<\tau(1), W\left(H_{\alpha}\right)=\alpha\right\}} \int_{0}^{\hat{T}_{-\alpha}} \mathbb{1}_{\{\hat{W}(u) \leq 0\}} d u .
\end{aligned}
$$

By the strong Markov property and symmetry, we obtain:

$$
\begin{aligned}
\mathbb{P}(X(1)>\lambda) & \geq \mathbb{P}\left(H_{\alpha}<\tau(1)\right) \mathbb{P}\left(\int_{0}^{T_{\alpha}} \mathbb{1}_{\{W(u) \geq 0\}} d u>\lambda\right) \\
& =\mathbb{P}\left(H_{\alpha}<\tau(1)\right) \mathbb{P}\left(\int_{0}^{T_{1}} \mathbb{1}_{\{W(u) \geq 0\}} d u>\frac{\lambda}{\alpha^{2}}\right),
\end{aligned}
$$

with $T_{x}=\inf \{t>0: W(t)=x\}$. According to the first Ray-Knight theorem (Revuz and Yor [6, Theorem XI.2.2]), $\left\{\ell\left(T_{1}, 1-x\right) ; 0 \leq x \leq 1\right\}$ is a two-dimensional square Bessel process (i.e. the square of Euclidean modulus of a two-dimensional Wiener process), starting from 0 . Since

$$
\int_{0}^{T_{1}} \mathbb{1}_{\{W(u) \geq 0\}} d u=\int_{0}^{1} \ell\left(T_{1}, 1-x\right) d x
$$

and since a two-dimensional square Bessel process is (stochastically) greater than the square of a real-valued Wiener process, we have

$$
\mathbb{P}(X(1)>\lambda) \geq \mathbb{P}\left(H_{\alpha}<\tau(1)\right) \mathbb{P}\left(\int_{0}^{1} W^{2}(u) d u>\frac{\lambda}{\alpha^{2}}\right) .
$$

Recall the Cameron-Martin formula ([1]):

$$
\mathbb{P}\left(\int_{0}^{1} W^{2}(u) d u>x\right) \sim \frac{\sqrt{32}}{\pi^{2}} x^{-1 / 2} \exp \left(-\frac{\pi^{2}}{8} x\right), \quad x \rightarrow \infty,
$$

(the symbol " $a(x) \sim b(x)$ " $\left(x \rightarrow x_{0}\right)$ denoting $\left.\lim _{x \rightarrow x_{0}} a(x) / b(x)=1\right)$. Consequently, for sufficiently large $\lambda$,

$$
\mathbb{P}(X(1)>\lambda) \geq \frac{C(\alpha)}{\lambda^{1 / 2}} \exp \left(-\frac{\pi^{2} \lambda}{8 \alpha^{2}}\right),
$$

with $C(\alpha)>0$ a finite constant depending only on $\alpha$. This implies the lower bound in Lemma 2.

Proof of Theorem 1. Let us begin with the upper bound. Fix a small $\varepsilon>0$. Define $t_{n}=(1+\varepsilon)^{n}$. Then $(1+5 \varepsilon) t_{n}^{2} / t_{n+1}^{2} \geq 1+2 \varepsilon$. By scaling property (2.1), we have

$$
\begin{aligned}
& \mathbb{P}\left(X\left(t_{n+1}\right)>(1+5 \varepsilon) \frac{8 \alpha^{2}}{\pi^{2}} t_{n}^{2} \log \log t_{n}\right) \\
& \quad=\mathbb{P}\left(X(1)>(1+5 \varepsilon) \frac{8 \alpha^{2}}{\pi^{2}} \frac{t_{n}^{2}}{t_{n+1}^{2}} \log \log t_{n}\right) \\
& \quad \leq \mathbb{P}\left(X(1)>(1+2 \varepsilon) \frac{8 \alpha^{2}}{\pi^{2}} \log \log t_{n}\right) .
\end{aligned}
$$


Using (2.3), the above expression is, when $n$ is sufficiently large, bounded above by

$$
\exp \left(-(1+\varepsilon) \log \log t_{n}\right)=\frac{1}{n^{1+\varepsilon}(\log (1+\varepsilon))^{1+\varepsilon}}
$$

which sums for $n$. It follows from the Borel-Cantelli lemma that (almost surely) for large $n, X\left(t_{n+1}\right) \leq(1+5 \varepsilon)\left(8 \alpha^{2} / \pi^{2}\right) t_{n}^{2} \log \log t_{n}$. Let $t \in\left[t_{n}, t_{n+1}\right]$. Then

$$
\frac{X(t)}{t^{2} \log \log t} \leq \frac{X\left(t_{n+1}\right)}{t_{n}^{2} \log \log t_{n}} \leq(1+5 \varepsilon) \frac{8 \alpha^{2}}{\pi^{2}}
$$

Accordingly,

$$
\limsup _{t \rightarrow \infty} \frac{X(t)}{t^{2} \log \log t} \leq(1+5 \varepsilon) \frac{8 \alpha^{2}}{\pi^{2}},
$$

for any $\varepsilon>0$. Letting $\varepsilon$ tend to $0^{+}$gives the upper bound in Theorem 1 . It remains to verify the lower bound part. Fix again an $\varepsilon>0$. Let $t_{n}=2^{n}$ and let

$$
A_{n}=\left\{\int_{\tau\left(t_{n-1}\right)}^{\tau\left(t_{n}\right)} \mathbb{1}_{\left\{|W(u)| \leq \alpha t_{n}\right\}} d u>(1-\varepsilon) \frac{8 \alpha^{2}}{\pi^{2}} t_{n}^{2} \log \log t_{n}\right\} .
$$

The measurable events $\left(A_{n}\right)$ are obviously independent. Moreover,

$$
\mathbb{P}\left(A_{n}\right)=\mathbb{P}\left(\int_{0}^{\tau\left(t_{n}-t_{n-1}\right)} \mathbb{1}_{\left\{|W(u)| \leq \alpha t_{n}\right\}} d u>(1-\varepsilon) \frac{8 \alpha^{2}}{\pi^{2}} t_{n}^{2} \log \log t_{n}\right),
$$

using the strong Markov property. By scaling, we have

$$
\begin{aligned}
\mathbb{P}\left(A_{n}\right) & =\mathbb{P}\left(\int_{0}^{\tau(1)} \mathbb{1}_{\left\{|W(u)| \leq \alpha t_{n} /\left(t_{n}-t_{n-1}\right)\right\}} d u>(1-\varepsilon) \frac{8 \alpha^{2}}{\pi^{2}} \frac{t_{n}^{2}}{\left(t_{n}-t_{n-1}\right)^{2}} \log \log t_{n}\right) \\
& =\mathbb{P}\left(\int_{0}^{\tau(1)} \mathbb{1}_{\{|W(u)| \leq 2 \alpha\}} d u>(1-\varepsilon) \frac{32 \alpha^{2}}{\pi^{2}} \log \log t_{n}\right),
\end{aligned}
$$

using the fact that $t_{n} /\left(t_{n}-t_{n-1}\right)=2$. Applying (2.3) to $2 \alpha$ (instead of $\alpha$ ) implies that for large $n$,

$$
\mathbb{P}\left(A_{n}\right) \geq \exp \left(-\log \log t_{n}\right)=\frac{1}{n \log 2},
$$

which forms a divergent series. Since the $A_{n}$ 's are independent, the Borel-Cantelli lemma tells $\mathbb{P}\left(A_{n}\right.$, i.o. $)=1$. Therefore

$$
\liminf _{t \rightarrow \infty} \frac{X(t)}{t^{2} \log \log t} \geq(1-\varepsilon) \frac{8 \alpha^{2}}{\pi^{2}} \quad \text { a.s. }
$$

Since the constant $\varepsilon>0$ can be arbitrarily small, this yields the lower bound in Theorem 1. 


\section{Proof of Theorem 2}

Let us keep the notation introduced previously. The first step is to establish the lower tail of $X(1)$.

Lemma 3. We have, for any $\alpha>0$,

$$
\mathbb{P}(X(1)<x) \sim\left(\frac{2}{\pi}\right)^{1 / 2} x^{1 / 2} \exp \left(-\frac{1}{2 x}\right), \quad x \rightarrow 0 .
$$

Consequently, there exists a finite constant $C>0$ such that

$$
\mathbb{P}(X(1)<x) \leq C \exp \left(-\frac{1}{2 x}\right), \quad \forall x>0 .
$$

The proof of Lemma 3 is based on a general complex analysis argument I have learnt from Csáki [2, p. 210], which is stated as follows.

Lemma 4. Let $g(z)$ be holomorphic on $\left\{(\operatorname{Im}(z))^{2}>4 \varepsilon(\varepsilon-\operatorname{Re}(z))\right\}$ for any suffciently small $\varepsilon>0$, and let $G(z)=g\left(z^{2}\right)$. Assume that for $t \rightarrow \infty$ there exist finite constants $\beta \geq 0, \gamma>1$ and $\eta>1 / 2$ such that

$$
\begin{aligned}
& G(t+i u)=o\left(G(t) \exp \left(\beta|u| t^{-1 / 2}\right)\right) \quad \text { uniformly for }|u| \geq t^{\eta}, \\
& G^{\prime \prime}(t+i u)=o\left(t^{-\gamma} G(t)\right) \quad \text { uniformly for }|u| \leq t^{\eta} .
\end{aligned}
$$

Then for any $c>0$,

$$
\frac{1}{2 \pi i} \int_{c-i \infty}^{c+i \infty} p^{-1 / 2} g(p) \exp \left(p s-2 p^{1 / 2}\right) d p=\frac{1+o\left(s^{(\gamma-1) / 2}\right)}{(\pi s)^{1 / 2}} g\left(s^{-2}\right) \exp \left(-\frac{1}{s}\right),
$$

as $s \rightarrow 0^{+}$.

Proof of Lemma 3. From (2.2), we have, using integration by parts,

$$
\int_{0}^{\infty} d x e^{-\theta x} \mathbb{P}(X(1)<x)=\frac{1}{\theta} \exp \left(-(2 \theta)^{1 / 2} \tanh \left(\alpha(2 \theta)^{1 / 2}\right)\right),
$$

for $\theta>0$. Inverting the Laplace transform yields

$$
\begin{aligned}
\mathbb{P}(X(1)<x) & =\frac{1}{2 \pi i} \int_{2 c-i \infty}^{2 c+i \infty} \frac{d \theta}{\theta} e^{x \theta} \exp \left(-(2 \theta)^{1 / 2} \tanh \left(\alpha(2 \theta)^{1 / 2}\right)\right) \\
& =\frac{1}{2 \pi i} \int_{c-i \infty}^{c+i \infty} \frac{d p}{p} e^{2 p x} \exp \left(-2 p^{1 / 2} \tanh \left(2 \alpha p^{1 / 2}\right)\right) \\
& =\frac{1}{2 \pi i} \int_{c-i \infty}^{c+i \infty} p^{-1 / 2} g(p) \exp \left(2 p x-2 p^{1 / 2}\right) d p,
\end{aligned}
$$

where

$$
g(p)=p^{-1 / 2} \exp \left(2 p^{1 / 2}-2 p^{1 / 2} \tanh \left(2 \alpha p^{1 / 2}\right)\right) .
$$

Obviously, $g(z)$ is holomorphic on $\left\{(\operatorname{Im}(z))^{2}>4 \varepsilon(\varepsilon-\operatorname{Re}(z))\right\}$ for any $\varepsilon>0$. Let

$$
G(z)=g\left(z^{2}\right)=\frac{1}{z} \exp (2 z-2 z \tanh (2 \alpha z))=\frac{1}{z} \exp \left(\frac{4 z}{e^{4 \alpha z}+1}\right) .
$$


We now verify conditions (3.3) and (3.4) for $G$. Write $G(z)=z^{-1} \exp (H(z))$, with $H(z) \equiv 4 z /\left(e^{4 \alpha z}+1\right)$. Then

$$
\begin{aligned}
\operatorname{Re}(H(t+i u))-H(t) & \leq|H(t+i u)-H(t)| \\
& =\frac{\left|4 t e^{4 \alpha t}\left(1-e^{4 i \alpha u}\right)+4 i u\left(e^{4 \alpha t}+1\right)\right|}{\left|e^{4 \alpha(t+i u)}+1\right|\left(e^{4 \alpha t}+1\right)} \\
& \leq \frac{8 t+4|u|}{e^{4 \alpha t}-1} .
\end{aligned}
$$

Since $(8 t+4|u|) /\left(e^{4 \alpha t}-1\right) \leq|u| t^{-1 / 2}$ for any $|u| \geq t \geq t_{0}\left(t_{0}\right.$ depending on the value of $\alpha$ ), the above inequality trivially implies (3.3) with $\beta=1$ and $\eta=1$. For (3.4), observe that $G^{\prime \prime}(z)=z^{-2}\left(2-2 z H^{\prime}(z)+z^{2} H^{\prime \prime}(z)+z^{2}\left(H^{\prime}(z)\right)^{2}\right) G(z)$. Assume $|u| \leq t$ and $t \geq t_{0}$. By (3.7), we have $\operatorname{Re}(H(t+i u))-H(t) \leq 1$. Thus $|G(t+i u)| \leq 3 G(t)$. Several lines of elementary calculation show that

$$
\left|2-2(t+i u) H^{\prime}(t+i u)+(t+i u)^{2} H^{\prime \prime}(t+i u)+(t+i u)^{2}\left(H^{\prime}(t+i u)\right)^{2}\right| \leq 3 .
$$

Consequently, (3.4) holds with $\gamma=3 / 2$ (actually any constant strictly smaller than 2 will do). Applying (3.5) to $s=2 x$ and using (3.6) completes the proof of Lemma 3 .

Proof of Theorem 2. The convergent half is an easy consequence of Lemma 3. Indeed, assume $f$ to be non-decreasing such that

$$
\int^{\infty} \frac{d t}{t} f^{1 / 2}(t) e^{-f(t) / 2}<\infty
$$

Thus $f(t) \uparrow \infty$ as $t \uparrow \infty$. Pick a large initial value $t_{0}$ and define the sequence $\left(t_{n}\right)$ by recurrence: $t_{n+1}=t_{n}\left(1+1 / f\left(t_{n}\right)\right)$ for $n=0,1,2, \cdots$. Obviously $\left(t_{n}\right)$ increases to infinity. A standard argument shows that (3.8) implies

$$
\sum_{n} f^{-1 / 2}\left(t_{n}\right) e^{-f\left(t_{n}\right) / 2}<\infty
$$

By scaling property (2.1) and small deviation probability estimate (3.1), we have

$$
\begin{aligned}
\mathbb{P}\left(X\left(t_{n}\right)<\frac{t_{n}^{2}}{f\left(t_{n}\right)-3}\right) & =\mathbb{P}\left(X(1)<\frac{1}{f\left(t_{n}\right)-3}\right) \\
& \leq\left(f\left(t_{n}\right)-3\right)^{-1 / 2} \exp \left(-\frac{f\left(t_{n}\right)-3}{2}\right) \\
& \leq 5 f^{-1 / 2}\left(t_{n}\right) e^{-f\left(t_{n}\right) / 2}
\end{aligned}
$$

which, according to (3.9), is summable. Applying the Borel-Cantelli lemma gives that (almost surely) for sufficiently large $n, X\left(t_{n}\right) \geq t_{n}^{2} /\left(f\left(t_{n}\right)-3\right)$. Let $t \in$ $\left[t_{n}, t_{n+1}\right]$. Then

$$
X(t) \geq X\left(t_{n}\right) \geq \frac{t_{n}^{2}}{f\left(t_{n}\right)-3}=\frac{t_{n+1}^{2}}{\left(1+1 / f\left(t_{n}\right)\right)^{2}\left(f\left(t_{n}\right)-3\right)} \geq \frac{t_{n+1}^{2}}{f\left(t_{n}\right)} \geq \frac{t^{2}}{f(t)},
$$


implying the convergent half of Theorem 1 . To check the divergent half, let $f>0$ be non-decreasing with

$$
\int^{\infty} \frac{d t}{t} f^{1 / 2}(t) e^{-f(t) / 2}=\infty
$$

In light of (1.4), let us assume without loss of generality that

$$
\log \log t \leq f(t) \leq 3 \log \log t
$$

For an elegant argument justifying (3.11), we refer to Csáki [2]. Let $\rho>0$ and $\varepsilon>0$. Fix a large initial value $i_{0} \equiv i_{0}(\rho, \varepsilon)$. Define $t_{i}=\exp (\rho i / \log i)\left(\right.$ for $\left.i \geq i_{0}\right)$. By means of (3.11), we have

$$
\begin{aligned}
& 1+\frac{\rho}{2 \log i} \leq \frac{t_{i}}{t_{i-1}} \leq 1+\frac{2 \rho}{\log i}, \\
& \frac{1}{2} \log i \leq f\left(t_{i}\right) \leq 3 \log i, \quad i>i_{0} .
\end{aligned}
$$

Moreover, it is easily seen from (3.10) that $\sum_{i} f^{-1 / 2}\left(t_{i}\right) \exp \left(-f\left(t_{i}\right) / 2\right)=\infty$. Now consider

$$
A_{i}=\left\{\frac{t_{i-1}^{2}}{f\left(t_{i}\right)} \leq X\left(t_{i}\right)<\frac{t_{i}^{2}}{f\left(t_{i}\right)}\right\}
$$

Using (2.1), (3.1), (3.12) and (3.13) gives

$$
\begin{aligned}
\mathbb{P}\left(A_{i}\right) & =\mathbb{P}\left[\frac{t_{i-1}^{2}}{t_{i}^{2} f\left(t_{i}\right)} \leq X(1)<\frac{1}{f\left(t_{i}\right)}\right] \\
& \geq(1-\varepsilon)\left(\frac{2}{\pi}\right)^{1 / 2}\left(f^{-1 / 2}\left(t_{i}\right) e^{-f\left(t_{i}\right) / 2}-\left(\frac{t_{i-1}^{2}}{t_{i}^{2} f\left(t_{i}\right)}\right)^{1 / 2} \exp \left(-\frac{t_{i}^{2} f\left(t_{i}\right)}{2 t_{i-1}^{2}}\right)\right) \\
& \geq(1-\varepsilon)\left(1-e^{-\rho / 4}\right)(2 / \pi)^{1 / 2} f^{-1 / 2}\left(t_{i}\right) e^{-f\left(t_{i}\right) / 2},
\end{aligned}
$$

for any $n>i_{0}$. Consequently,

$$
\sum_{i} \mathbb{P}\left(A_{i}\right)=\infty
$$

Pick $i_{0}<i<j$. In view of the strong Markov property, we have

$$
\begin{aligned}
\mathbb{P}\left(A_{i} A_{j}\right) & \leq \mathbb{P}\left(A_{i}, \int_{\tau\left(t_{i}\right)}^{\tau\left(t_{j}\right)} \mathbb{1}_{\left\{|W(u)| \leq \alpha t_{j}\right\}} d u<\frac{t_{j}^{2}}{f\left(t_{j}\right)}-\frac{t_{i-1}^{2}}{f\left(t_{i}\right)}\right) \\
& \leq \mathbb{P}\left(A_{i}\right) \mathbb{P}\left(\int_{0}^{\tau\left(t_{j}-t_{i}\right)} \mathbb{1}_{\left\{|W(u)| \leq \alpha\left(t_{j}-t_{i}\right)\right\}} d u<\frac{t_{j}^{2}-t_{i-1}^{2}}{f\left(t_{j}\right)}\right) .
\end{aligned}
$$

The above estimate together with scaling property (2.1) readily yield

$$
\mathbb{P}\left(A_{i} A_{j}\right) \leq \mathbb{P}\left(A_{i}\right) \mathbb{P}\left(X(1)<\frac{t_{j}^{2}-t_{i-1}^{2}}{\left(t_{j}-t_{i}\right)^{2} f\left(t_{j}\right)}\right),
$$


for any $i_{0}<i<j$. Define

$$
\begin{aligned}
& \mathcal{E}(n)=\left\{i_{0}<i<j \leq n: j-i<(\log i)^{3}\right\} \\
& \mathcal{F}(n)=\left\{i_{0}<i<j \leq n: j-i \geq(\log i)^{3}\right\} .
\end{aligned}
$$

Remark that when $i<j<i+(\log i)^{3}$,

$$
\frac{j}{\log j}-\frac{i}{\log i}=\frac{(j-i) \log i-i \log (1+(j-i) / i)}{\log i \log j} \sim \frac{j-i}{\log j} \sim \frac{j-i}{\log i},
$$

as $i \rightarrow \infty$. Let $(i, j) \in \mathcal{E}(n)$. Then by the above observation, we have

$$
\exp \left(-\frac{2 \rho(j-i)}{\log i}\right) \leq \frac{t_{i-1}}{t_{j}} \leq \frac{t_{i}}{t_{j}} \leq \exp \left(-\frac{\rho(j-i)}{2 \log i}\right) .
$$

Therefore by (3.13),

$$
\begin{aligned}
\frac{t_{j}^{2}-t_{i-1}^{2}}{\left(t_{j}-t_{i}\right)^{2} f\left(t_{j}\right)} & \leq \frac{t_{j}^{2}-t_{i-1}^{2}}{\left(t_{j}-t_{i}\right)^{2} f\left(t_{i}\right)} \leq \frac{1-\exp (-4 \rho(j-i) / \log i)}{(1-\exp (-\rho(j-i) / 2 \log i))^{2}} \frac{2}{\log i} \\
& \leq \frac{C_{1}}{\min (j-i, \log i)},
\end{aligned}
$$

for some finite constant $C_{1}>0$ depending only on $\rho$ and $\varepsilon$ ( $i_{0}$ depending on $\varepsilon$ ). Using (3.16) and (3.2), we obtain (writing $C_{2} \equiv 1 /\left(2 C_{1}\right)$ in the sequel):

$$
\begin{aligned}
\mathbb{P}\left(A_{i} A_{j}\right) & \leq C \mathbb{P}\left(A_{i}\right) \exp \left(-C_{2} \min (j-i, \log i)\right) \\
& \leq C \mathbb{P}\left(A_{i}\right) \exp \left(-C_{2}(j-i)\right)+C \mathbb{P}\left(A_{i}\right) \exp \left(-C_{2} \log i\right),
\end{aligned}
$$

( $C$ being the constant introduced in (3.2)) which in turn implies

$$
\begin{aligned}
\sum_{(i, j) \in \mathcal{E}(n)} \sum_{i} \mathbb{P}\left(A_{i} A_{j}\right) \leq & C \sum_{i=i_{0}+1}^{n} \mathbb{P}\left(A_{i}\right) \sum_{j>i} e^{-C_{2}(j-i)} \\
& +C \sum_{i=i_{0}+1}^{n} \mathbb{P}\left(A_{i}\right) \sum_{i<j<i+(\log i)^{3}} i^{-C_{2}} \\
\leq & C_{3} \sum_{i=i_{0}+1}^{n} \mathbb{P}\left(A_{i}\right),
\end{aligned}
$$

for some finite constant $C_{3}>0$. Now let $(i, j) \in \mathcal{F}(n)$. In this case, $j-(\log j)^{2} \geq$ $i+(\log i)^{3}-\left(\log \left(i+(\log i)^{3}\right)\right)^{2} \geq i$. Thus $j-i \geq(\log j)^{2}$. It is noticed that

$$
\frac{j}{\log j}-\frac{i}{\log i}=\frac{(j-i) \log i-i \log (1+(j-i) / i)}{\log i \log j} \geq \frac{j-i}{2 \log j}
$$


Therefore, we have

$$
\begin{aligned}
\frac{t_{j}^{2}-t_{i-1}^{2}}{\left(t_{j}-t_{i}\right)^{2} f\left(t_{j}\right)} & \leq \frac{1}{\left(1-t_{i} / t_{j}\right)^{2} f\left(t_{j}\right)} \leq \frac{1}{(1-\exp (-\rho(j-i) / 2 \log j))^{2} f\left(t_{j}\right)} \\
& \leq \frac{1}{\left(1-(\log j)^{-2}\right) f\left(t_{j}\right)} .
\end{aligned}
$$

According to (3.16) and (3.1), we get

$$
\mathbb{P}\left(A_{i} A_{j}\right) \leq(1+\varepsilon) \mathbb{P}\left(A_{i}\right)\left(\frac{2}{\pi}\right)^{1 / 2} f^{-1 / 2}\left(t_{j}\right) \exp \left(-\frac{\left(1-(\log j)^{-2}\right) f\left(t_{j}\right)}{2}\right) .
$$

Since $(\log j)^{-2} f\left(t_{j}\right) / 2 \leq 3 / 2 \log j \leq \varepsilon$ for $j \geq i_{0}$, the above estimate together with (3.14) confirm

$$
\mathbb{P}\left(A_{i} A_{j}\right) \leq \frac{e^{\varepsilon}(1+\varepsilon)}{(1-\varepsilon)\left(1-e^{-\rho / 4}\right)} \mathbb{P}\left(A_{i}\right) \mathbb{P}\left(A_{j}\right),
$$

which, with the aid of (3.17) and (3.15), yields

$$
\liminf _{n \rightarrow \infty} \sum_{i=i_{0}+1}^{n} \sum_{j=i_{0}+1}^{n} \mathbb{P}\left(A_{i} A_{j}\right) /\left(\sum_{i=i_{0}+1}^{n} \mathbb{P}\left(A_{i}\right)\right)^{2} \leq \frac{1}{1-e^{-\rho / 4}} .
$$

Since $\sum_{i} \mathbb{P}\left(A_{i}\right)$ diverges, using a well-known version of the Borel-Cantelli lemma (see for example Révész $[5$, p. 28$]$ ) gives $\mathbb{P}\left(A_{i}\right.$, i.o. $) \geq 1-e^{-\rho / 4}$. A fortiori, we have

$$
\mathbb{P}\left[X(t)<\frac{t^{2}}{f(t)}, \text { i.o. }\right] \geq 1-e^{-\rho / 4},
$$

for any $\rho>0$. The proof of the divergent half is completed by sending $\rho$ to infinity.

\section{ACKNOWLEDGEMENTS}

I am grateful to Marc Yor for helpful comments on the first draft of this work. Many thanks are due to an anonymous referee for a careful reading of the manuscript and for valuable suggestions.

\section{REFERENCES}

1. R.H. Cameron and W.T. Martin, The Wiener measure of Hilbert neighborhoods in the space of real continuous functions, J. Math. Phys. 23 (1944), 195-209. MR 6:132a

2. E. Csáki, An integral test for the supremum of Wiener local time, Probab. Th. Rel. Fields $\mathbf{8 3}$ (1989), 207-217. MR 91a:60206

3. A. Földes and M.L. Puri, The time spent by the Wiener process in a narrow tube before leaving a wide tube, Proc. Amer. Math. Soc. 117 (1993), 529-536. MR 93d:60131

4. J.W. Pitman and M. Yor, A decomposition of Bessel bridges, Z. Wahrscheinlichkeitstheorie verw. Gebiete 59 (1982), 425-457. MR 84a:60091

5. P. Révész, Random Walk in Random and Non-Random Environments, World Scientific, Singapore, 1990. MR 92c:60096

6. D. Revuz and M. Yor, Continuous Martingales and Brownian Motion, 2nd ed., Springer, Berlin, 1994. CMP 95:04

7. H.F. Trotter, A property of Brownian motion paths, Illinois J. Math. 2 (1958), 425-433. MR 20:2795

Université Paris VI, L.S.T.A. - CNRS URA 1321, Université Paris VI, Tour 45-55, 4

Place Jussieu, F-75252 Paris Cedex 05, France

E-mail address: shi@ccr.jussieu.fr 